\title{
Secondhand Smoke Exposure in Household during Childhood
}

National Cancer Institute

\section{Source}

National Cancer Institute. Secondhand Smoke Exposure in Household during Childhood.

NCl Thesaurus. Code C156837.

Secondhand smoke exposure was present in the household during childhood. 